\title{
Treatment of non-radiographic axial spondyloarthritis: it is only the beginning
}

\author{
Irene E van der Horst-Bruinsma
}

The new nomenclature used in spondyloarthritis (SpA), axial versus peripheral, as proposed by the Assessment of SpondyloArthritis international Society (ASAS) and accepted in 2009, has changed the perspective of ankylosing spondylitis (AS) and other types of spondylarthropathies. ${ }^{1} 2$ The axial type of SpA is subdivided in the (early) nonradiographic type (nr-axial $\mathrm{SpA}$ ) and the radiographic type, which is equivalent to the diagnosis of AS (according to the modified New York criteria ${ }^{3}$ ). Instead of debating whether patients with AS do fulfil the grade of radiographic signs of sacroiliitis necessary for the diagnosis AS, the focus of interest has shifted towards the spectre of early detection of inflammation at the MRI of the pelvis and spine. The great advantage of the new nomenclature with an earlier phase of the disease is that the delay in diagnosis of $\mathrm{SpA}$ is shortened by years. Now the current research is directed towards the treatment options of this early stage.

The first question to be answered in the near future is whether early antitumor necrosis factor (TNF) treatment is effective on clinical symptoms in nr-axial SpA. The second question will be whether treatment in this early phase of the disease will sufficiently suppress inflammation in the long term. Third, and most importantly, the question arises whether this early treatment with anti-TNF has a positive benefit:risk ratio. This early and expensive treatment might diminish symptoms, but it is unclear whether it decreases or even halts the rate of radiographic progression. So far, there is no clear evidence that anti-TNF does delay this process in established AS.

In order to answer the first two questions, two interesting studies are published in Annals of Rheumatic Diseases. ${ }^{4} 10$

Correspondence to $\mathrm{Dr}$ Irene $\mathrm{E}$ van der HorstBruinsma, Department of Rheumatology, VU University Medical Center, Room 3A-64, P.O. Box 7057, Amsterdam 1007 MB, The Netherlands;

ie.vanderhorst@vumc.nl
The ABILITY trial compares the efficacy of adalimumab versus placebo in nr-axial SpA after 12 weeks of treatment. ${ }^{4}$ The outcome parameters used in this study were the ASAS $20 \%$ and $40 \%$ response, and level of inflammation was evaluated by CRP-levels and SPARCC MRI scores of the sacroiliac joints and spine. The results showed a significantly higher number of ASAS40 responders in the adalimumab group (36\%) compared with placebo $(15 \%)$, but the difference between number of responders to adalimumab and placebo is rather small, only $21 \%$. A same small difference $(21 \%$ ) was also observed between ASAS responders after 12 weeks: $52 \%$ to adalimumab and $31 \%$ to placebo. The relatively high number of responders in the placebo group (31\%), is higher compared with most AS studies (between 9\% and 21\% ASAS 20 responders. ${ }^{5-7}$ This high placebo response rate might be explained by the outcome parameter, the ASAS response, which is based on patient questionnaires for AS and not validated for the nr-axial SpA population, as the authors already mention in their discussion. Part of the response could also be explained by regression to the mean after inclusion at a high level of disease activity.

The difference in ASDAS response, though, which also includes the objective parameter CRP, is not very high, but nevertheless, significant (the difference in mean ASDAS change between placebo and adalimumab is -0.7 after 12 weeks).

Subanalyses of the patients achieving ASAS 40 response show a trend towards a higher responsiveness in case of elevated CRP, younger age (below 40 years) and higher SPARCC scores at the MRI of the sacroiliac joint (SI) joints, but the number of patients in these subgroups is very small. Moreover, in patients over 40 years of age the percentage of ASAS40 responders is only $17 \%$ versus $20 \%$ in the placebo and adalimumab groups, respectively, so these patients might not benefit much from this therapy, and larger studies are needed to explore this. By contrast, in patients with a negative MRI and a normal CRP-level at baseline, the response rates between both groups was almost similar, $23 \%$ in the adalimumab and $20 \%$ in the placebo group. However, the number of patients in these subgroups of ASAS40 responders (figure 2) $(5 / 22$ in adalimumab vs $4 / 20)$ in placebo) is far too small to draw any conclusions, and this issue should be explored in larger studies.

A few interesting points in the patient selection of this study are the exclusion of patients with psoriasis and the relatively high number of patients who also suffered from arthritis (35-52\%) compared with other AS populations, in which peripheral arthritis occurs in $25 \%{ }^{8}$

Interestingly, $18 \%$ of the patients use disease modifying antireumatic drugs (DMARDs) which might influence the joint count and CRP levels (and the ASDAS score as well), because the number of concomitant DMARD users is higher in the MRI-HLA-B27+ subgroup $(27 \%)$ treated with adalimumab compared with the other groups (table 1). It was not described whether these DMARDs used include sulfasalazine, which does have efficacy in peripheral arthritis and might have a beneficial effect on spinal symptoms as well. ${ }^{9}$

Another study ${ }^{10}$ which focuses on the treatment of nr-axial SpA with another TNF blocker, etanercept, is a subanalysis of the ESTHER trial. This trial compares the efficacy of etanercept with sulfasalazine in nr-axial SpA and in early AS with a symptom duration not longer than 5 years. ${ }^{11}$ All patients in this trial had to have inflammatory lesions at the wholebody MRI. The number of nr-axial SpA patients with a negative MRI were not described in this paper but other studies report a high number, around 70\%. ${ }^{12} 13$ The conclusion of the ESTHER trial, involving the whole group of patients (AS and nr-axial spA) was that the efficacy of etanercept was much higher than of sulfasalazine, with an ASAS40 response of $70 \%$ in the etanercept compared with $31 \%$ in the placebo group.

A subanalysis in the ESTHER study ${ }^{10}$ was only made of the etanercept (and not the sulfasalazine)-treated patients, which showed equal responsiveness in the 20 AS patients and 20 nr-axial SpA patients, with an ASAS40 response of $75 \%$ versus $65 \%$, respectively (table 2 ).

Comparison of both studies in nrradiographic $\mathrm{SpA}$ shows a much higher ASAS40 response due to etanercept (ESTHER study) compared with the adalimumab (ABILITY trial). This difference 
in response can be explained by another method of patient selection in the ESTHER study, because active inflammation of the spine and/or sacroiliac joints on whole-body MRI was mandatory. As discussed before, a positive MRI is one of the predictors of good response in AS as well as in nr-axial SpA. Unfortunately, the definition of a positive MRI differs among studies, because in the ESTHER trial, a whole-body MRI was used, and in other studies only SI joints.

The ASAS40 response of $65 \%$ in the etanercept nr-axial SpA group $(n=20)$ seems higher compared with the $41 \%$ found in the MRI-positive/or elevated CRP group treated with adalimumab $(n=28 / 69)$, but inclusion criteria differ too much and the numbers are too small to draw any final conclusions.

Another difference between these studies is the treatment period, which was much longer in the etanercepttreated patients (48 weeks), compared with the adalimumab study (12 weeks). It is not clear whether a longer treatment period with anti-TNF might have a different effect at the level of inflammation and outcome of long-term nr-axial SpA.

It would be very interesting to know whether sulfasalazine, which is much cheaper than a TNF blocker, would be effective in nr-axial SpA as well, but unfortunately this subanalysis was not performed. The ESTHER trial (which includes $51 \%$ AS patients and $49 \%$ of nr-axial SpA) shows in the sulfasalazinetreated patients an ASAS20 response of $42 \%$ and ASAS40 of $31 \%$, which is relatively high. The MRI score also improves in the sulfasalazine group (35\%) but much less compared with the etanercepttreated patients (almost 70\%).

In conclusion, treatment with nr-axial SpA with etanercept and adalimumab is effective, but so far does not show as impressive response rates as observed in AS. More studies are needed to define the characteristics of the patients that will benefit most from this treatment. So far, an elevated CRP and/or positive MRI of the SI joints or spine, seem to be the best predictors for clinical response to anti-TNF. However, the number of nr-axial SpA patients who have these characteristics is rather small, because positive MRIs of the SI joints was only found in $25-30 \%$ of the cases ${ }^{8}{ }^{9}$ which raises the question whether a positive MRI is prevalent enough in nr-axial SpA to be used for this selection. Moreover, it is not clear yet whether nr-axial SpA patients who have a raised CRP and or positive MRI have a better outcome due to anti-TNF compared with patients without these characteristics.

Whether other, less expensive treatment options such as sulfasalazine, might be beneficial in the early stage of axial SpA is not clear yet. More studies in nr-axial patients with longer treatment periods are needed with TNF blockers, also, other drugs are needed to answer the remaining questions of efficacy in the long term, and the influence of early treatment on radiographic progression.

\section{Competing interests None.}

Provenance and peer review Not commissioned; externally peer reviewed.

Accepted 19 March 2013

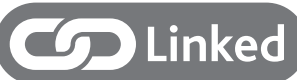

- http://dx.doi.org/10.1136/annrheumdis-2012-201766 http://dx.doi.org/10.1136/annrheumdis-2012-202389

Ann Rheum Dis 2013;72:789-790.

doi:10.1136/annrheumdis-2012-202908

\section{REFERENCES}

1. Rudwaleit M, van der Heijde D, Landewé $\mathrm{R}$, et al The development of Assessment of SpondyloArthritis international Society classification criteria for axial spondyloarthritis (part II): validation and final selection. Ann Rheum Dis 2009;68:777-83.

2. Rudwaleit $\mathbf{M}$, van der Heijde D, Landewé $\mathrm{R}$, et al The Assessment of SpondyloArthritis International
Society classification criteria for peripheral spondyloarthritis and for spondyloarthritis in general. Ann Rheum Dis 2011;70:25-31.

3. Van der Linden $\mathbf{S}$, Valkenburg HA, Cats $A$. Evaluation of the diagnostic criteria for ankylosing spondylitis; a proposal for the modification of the New York criteria. Arthritis Rheum 1984;27: 361-8.

4. Sieper J, van der Heijde D, Dougados M, et al. Efficacy and safety of adalimumab in patients with non-radiographic Axial Spondyloarthritis: Results of a Randomised, Placebo-controlled Trial (ABILITY-1). Ann Rheum Dis Published Online First 7 Jul 2012. doi:10. 1136/annrheumdis-2012-201766

5. Braun J, Brandt J, Listing J, et al. Treatment of active ankylosing spondylitis with infliximab-a double-blind, placebo-controlled multicenter trial. Lancet 2002;359:1187-93.

6. Brandt J, Khariouzov A, Listing J, et al. Successful short term treatment of patients with severe undifferentiated spondyloarthritis with the anti-tumor necrosis factor-alpha fusion receptor protein etanercept. J Rheumatol 2004;31:531-8.

7. vd Heyde D, Schiff M, Sieper J, et al. Adalimumab effectiveness for the treatment of ankylosing spondylitis is maintained for up to two years. Ann Rheum Dis 2009;68:922-9.

8. Khan MA. Ankylosing spondylitis: clinical features. In: JH Klippel, PA Dieppe, eds. Rheumatology, Vol. 2. London, UK: Mosby, 2000;16.1-16.10.

9. Braun J, van der Horst-Bruinsma IE, Huang $F$, et al. Clinical efficacy and safety of etanercept versus sulfasalazine in patients with ankylosing spondylitis: a randomized, double-blind trial. Arthritis Rheum 2011;63:1543-51.

10. Song $\mathbf{I}-\mathbf{H}$, Weiss A, Hermann K-G, et al. Similar response rates in patients with ankylosing spondylitis and non-radiographic axial spondyloarthritis after one year of treatment with etanercept-results from the ESTHER trial. Ann Rheum Dis 2013;72: 823-5.

11. Song IH, Hermann $\mathrm{K}$, Haibel $\mathrm{H}$, et al. Effects of etanercept versus sulfasalazine in early axial spondyloarthritis on active inflammatory lesions as detected by whole-body MRI (ESTHER): a 48-week randomised controlled trial. Ann Rheum Dis 2011;70:590-6. Erratum in: Ann Rheum Dis 2011;70(7):1350.

12. van der Horst-Bruinsma IE, van $\operatorname{der}$ Weijden $M$, Bruijnen S, et al, Low percentage of MRI changes in clinically suspected axial spondyloarthritis. Ann Rheum Dis 2012;71(Suppl 3):689.

13. de Hooge $\mathbf{M}$, van den Berg $R$, Navarro-Compán V, et al. Magnetic resonance imaging of the sacroiliac joints in the early detection of spondyloarthritis: no added value of gadolinium compared with short tau inversion recovery sequence. Rheumatology (Oxford) Published Online First: 16 Feb 2013. doi: 10.1093/ rheumatology/ket012. 